Int. J. Contemp. Math. Sciences, Vol. 2, 2007, no. 26, 1293 - 1305

\title{
On the variety of two dimensional real associative algebras
}

\author{
José María Ancochea Bermúdez ${ }^{1}$ \\ Dpto. Geometría y Topología. Facultad de Matemáticas \\ Universidad Complutense de Madrid \\ Plaza de Ciencias, 328040 Madrid, Spain \\ ancochea@mat.ucm.es \\ Javier Fresán \\ Dpto. Geometría y Topología. Facultad de Matemáticas \\ Universidad Complutense de Madrid \\ Plaza de Ciencias, 328040 Madrid, Spain \\ jfresan@estumail.ucm.es \\ Jonathan Sánchez Hernández \\ Dpto. Geometría y Topología. Facultad de Matemáticas \\ Universidad Complutense de Madrid \\ Plaza de Ciencias, 328040 Madrid, Spain \\ jnsanchez@mat.ucm.es
}

\begin{abstract}
This paper consists of a description of the variety of two dimensional associative algebras within the framework of Nonstandard Analysis. By decomposing each algebra in $A^{2}$ as sum of a Jordan algebra and a Lie algebra, we calculate the isomorphism classes of two dimensional associative algebras over the field of real numbers and determine the open components and the contractions of the variety.
\end{abstract}

Mathematics Subject Classification: 16B99

Keywords: associative algebras, rigidity, contraction

\footnotetext{
${ }^{1}$ This author has been partially supported by the research proyect MTM2006-09152 of Ministerio de Educación y Ciencia
} 


\section{Introduction}

The aim of this work is to study some properties of the variety of two dimensional associative algebras, specially those concerning rigidity and contractions. We first obtain a decomposition of associative algebras as sum of a Jordan algebra and a Lie algebra, which enables us to use known results on Jordan algebras [1] to classify two dimensional associative algebras over the field of real numbers. Then we introduce the concept of perturbation within Nelson's Internal Set Theory [6] and derive the perturbation equations of the variety. Nonstandard Analysis tools permit us to prove that $\mathcal{A}^{2}$ has four open components, two of dimension 4 and two of dimension 2. The remaining algebras of the variety are obtained by contraction of the rigid algebras which define the open components.

Definition 1. An associative algebra law over $\mathbb{R}$ is a bilinear mapping $\beta: \mathbb{R}^{n} \times \mathbb{R}^{n} \longrightarrow \mathbb{R}^{n}$ satisfying the constraint

$$
\beta(\beta(x, y), z)-\beta(x, \beta(y, z))=0, \quad x, y, z \in \mathbb{R}^{n} .
$$

We will abbreviate $\beta(x, y)$ by $x \circ y$, and $\mathcal{A}^{n}$ will denote the set of associative algebras over $\mathbb{R}^{n}$. The ordered pair $\left(\mathbb{R}^{n}, \beta\right)$ is called associative algebra.

Definition 2. A Jordan algebra law over $\mathbb{R}$ is a symmetric bilinear mapping $\varphi: \mathbb{R}^{n} \times \mathbb{R}^{n} \longrightarrow \mathbb{R}^{n}$ which verifies the identity

$$
\varphi(\varphi(x, x), \varphi(x, y))-\varphi(x, \varphi(\varphi(x, x), y))=0, \quad x, y \in \mathbb{R}^{n} .
$$

The ordered pair $\left(\mathbb{R}^{n}, \varphi\right)$ is a Jordan algebra and $\mathcal{J}^{n}$ will denote the set of Jordan algebra laws.

Definition 3. A Lie algebra law over $\mathbb{R}^{n}$ is an alternate bilinear mapping $\mu: \mathbb{R}^{n} \times \mathbb{R}^{n} \longrightarrow \mathbb{R}^{n}$ satisfying the Jacobi identity

$$
\mu(\mu(x, y), z)+\mu(\mu(y, z), x)+\mu(\mu(z, x), y)=0, \quad x, y, z \in \mathbb{R}^{n} .
$$

We will denote the set of Lie algebras over $\mathbb{R}^{n}$ by $\mathcal{L}^{n}$. The ordered pair $\left(\mathbb{R}^{n}, \mu\right)$, where $\mu \in \mathcal{L}^{n}$, is a Lie algebra.

Now we enunciate a proposition which plays a fundamental role in the classification which will be given.

Proposition 1. Let $\left(\mathbb{R}^{n}, \circ\right)$ be a real associative algebra. Then:

1. The law $\varphi$ defined by

$$
\varphi(x, y)=\frac{x \circ y+y \circ x}{2}, \quad x, y \in \mathbb{R}^{n}
$$

is a Jordan algebra law. 
2. The law $\mu$ defined by

$$
\mu(x, y)=\frac{x \circ y-y \circ x}{2}, \quad x, y \in \mathbb{R}^{n}
$$

is a Lie algebra law.

Let us recall that if there exists a non-zero vector $u$ such that $\beta(u, v)=0$ for all $v \in \mathbb{R}^{n}$, we say that $\beta$ has left isotropy. Similarly, if $\beta(v, u)=0$ for all $v \in \mathbb{R}^{n}$, then $u$ is a right isotropic vector. An associative algebra is said to be simple if it does not admit any proper ideal.

Let $B=\left\{e_{1}, \ldots, e_{n}\right\}$ be a basis for $\mathbb{R}^{n}$. It is possible to identify each algebra in $\mathcal{A}^{n}$ with its structure constants, that is, to consider $\beta \in \mathcal{A}^{n}$ as the tensor $\left(a_{i j}^{k}\right) \in \mathbb{R}^{n^{3}}$ whose coordinates, univocally determined by $e_{i} \circ e_{j}=a_{i j}^{k} e_{k}$, are the solutions of the system

$$
a_{i j}^{h} a_{h k}^{l}=a_{i h}^{l} a_{j k}^{h}, \quad 1 \leq i, j, k, h, l \leq n
$$

That gives $\mathcal{A}^{n}$ a structure of algebraic variety embedded in $\mathbb{R}^{n^{3}}$. From now on we will identify each algebra with its law. In the case of associative algebras, non-written products will be supossed to be zero. If $\varphi$ is a Jordan algebra or a Lie algebra only non-zero products $\varphi\left(e_{i}, e_{j}\right)$, with $i \leq j$, will be written.

\section{Classification of two dimensional real asso- ciative algebras}

If $n=2$, real associative algebras are given by the relations

$$
\begin{aligned}
& e_{1} \circ e_{1}=a_{1} e_{1}+a_{2} e_{2}, \\
& e_{1} \circ e_{2}=b_{1} e_{1}+b_{2} e_{2}, \\
& e_{2} \circ e_{1}=c_{1} e_{1}+c_{2} e_{2}, \\
& e_{2} \circ e_{2}=d_{1} e_{1}+d_{2} e_{2} .
\end{aligned}
$$

Or equivalently by a coefficient matrix of the form:

$$
\left(\begin{array}{cc}
a_{1} & a_{2} \\
b_{1} & b_{2} \\
c_{1} & c_{2} \\
d_{1} & d_{2}
\end{array}\right)
$$


Developing (2) the following equations are obtained:

$$
\begin{aligned}
a_{2} b_{1} & =a_{2} c_{1}, \\
a_{2} b_{2} & =a_{2} c_{2}, \\
b_{1} b_{2} & =a_{2} d_{1}, \\
a_{2} d_{1} & =c_{1} c_{2}, \\
a_{2} b_{1}+b_{2}^{2} & =a_{1} b_{2}+a_{2} d_{2}, \\
a_{1} c_{1}+b_{1} c_{2} & =a_{1} b_{1}+b_{2} c_{1}, \\
a_{1} d_{1}+b_{1} d_{2} & =b_{1}^{2}+b_{2} d_{1}, \\
a_{1} c_{2}+a_{2} d_{2} & =a_{2} c_{1}+c_{2}^{2}, \\
b_{1} c_{2}+b_{2} d_{2} & =b_{2} c_{1}+c_{2} d_{2}, \\
c_{1}^{2}+c_{2} d_{1} & =a_{1} d_{1}+c_{1} d_{2} .
\end{aligned}
$$

Thus, $A^{2}$ is an algebraic variety embedded in $\mathbb{R}^{8}$ and defined by the above system of homogeneous polynomials.

Let us consider the natural action of the general linear group $G L(n, \mathbb{R})$ over the variety:

$$
\begin{aligned}
G L(n, \mathbb{R}) \times \mathcal{A}^{n} & \longrightarrow \mathcal{A}^{n} \\
(f, \beta) & \longmapsto f^{-1} * \beta *(f \times f),
\end{aligned}
$$

with $f^{-1} * \beta *(f \times f)(x, y)=f^{-1}\left(\beta(f(x), f(y))\right.$. For each $\beta \in \mathcal{A}^{n}$, the orbit under this action, $\mathcal{O}(\beta)$, represents the set of associative algebras isomorphic to $\beta$. Our first aim is to determine the space of orbits

$$
A^{2} / G L(2, \mathbb{R})=\{\mathcal{O}(\beta)\}_{\beta \in \mathcal{A}^{2}}
$$

that is, the isomorphism classes of two dimensional real associative algebras.

For that purpose, we will make use of the decomposition

$$
x \circ y=\frac{x \circ y+y \circ x}{2}+\frac{x \circ y-y \circ x}{2}=\varphi(x, y)+\mu(x, y),
$$

where $\varphi$ is a Jordan algebra and $\mu$ is a Lie algebra defined by the product $\mu\left(e_{1}, e_{2}\right)=a e_{1}+b e_{2}$. In [1] a classification theorem for $\mathcal{J}^{2}$ is proved:

Theorem 1. Let $\varphi$ be a non-Abelian two dimensional real Jordan algebra. Then $\varphi$ is isomorphic to one of the following pairwise non-isomorphic Jordan algebras:

1. $\varphi_{1}\left(e_{1}, e_{1}\right)=e_{1}, \varphi_{1}\left(e_{1}, e_{2}\right)=e_{2}, \varphi_{1}\left(e_{2}, e_{2}\right)=-e_{1}$.

2. $\varphi_{2}\left(e_{1}, e_{1}\right)=e_{1}, \varphi_{2}\left(e_{1}, e_{2}\right)=e_{2}, \varphi_{2}\left(e_{2}, e_{2}\right)=e_{1}$.

3. $\varphi_{3}\left(e_{1}, e_{1}\right)=e_{1}, \varphi_{3}\left(e_{1}, e_{2}\right)=e_{2}, \varphi_{3}\left(e_{2}, e_{2}\right)=0$. 
On the variety of two dimensional real associative algebras

4. $\varphi_{4}\left(e_{1}, e_{1}\right)=0, \quad \varphi_{4}\left(e_{1}, e_{2}\right)=0, \quad \varphi_{4}\left(e_{2}, e_{2}\right)=e_{2}$.

5. $\varphi_{5}\left(e_{1}, e_{1}\right)=e_{2}, \varphi_{5}\left(e_{1}, e_{2}\right)=0, \quad \varphi_{5}\left(e_{2}, e_{2}\right)=0$.

6. $\varphi_{6}\left(e_{1}, e_{1}\right)=e_{1}, \varphi_{6}\left(e_{1}, e_{2}\right)=\frac{1}{2} e_{2}, \varphi_{6}\left(e_{2}, e_{2}\right)=0$.

Example 1. To illustrate how this result may be applied to solve the classification problem, let us consider the case in which $\varphi$ is isomorphic to $\varphi_{1}$. Then, there exists a basis $\left\{e_{1}, e_{2}\right\}$ such that:

$$
\begin{aligned}
& e_{1} \circ e_{1}=\varphi_{1}\left(e_{1}, e_{1}\right)+\mu\left(e_{1}, e_{1}\right)=e_{1} . \\
& e_{1} \circ e_{2}=\varphi_{1}\left(e_{1}, e_{2}\right)+\mu\left(e_{1}, e_{2}\right)=a e_{1}+(1+b) e_{2} . \\
& e_{2} \circ e_{1}=\varphi_{1}\left(e_{2}, e_{2}\right)+\mu\left(e_{2}, e_{1}\right)=-a e_{1}+(1-b) e_{2} . \\
& e_{2} \circ e_{2}=\varphi_{1}\left(e_{2}, e_{2}\right)+\mu\left(e_{2}, e_{2}\right)=-e_{1} .
\end{aligned}
$$

Structure constants must satisfy (3), so $a=b=0$ and $\beta$ coincides with $\varphi_{1}$.

Making the same calculus for each $\varphi_{i} \in \mathcal{J}^{2}$, the following theorem is obtained. For the first five Jordan algebras we have $a=b=0$, but when we consider $\varphi$ isomorphic to $\varphi_{6}$, the system (3) admits two different solutions: $\left(0, \frac{1}{2}\right)$ and $\left(0,-\frac{1}{2}\right)$. Thus, there are seven isomorphism classes in $\mathcal{A}^{2}$.

Theorem 2. Let $\beta$ be a two dimensional real associative algebra. If $\beta$ is not Abelian, then $\beta$ is isomorphic to one of the following pairwise non-isomorphic associative algebras:

1. $\beta_{1}: e_{1} \circ e_{1}=e_{1}, e_{1} \circ e_{2}=e_{2}, e_{2} \circ e_{1}=e_{2}, e_{2} \circ e_{2}=-e_{1}$.

2. $\beta_{2}: e_{1} \circ e_{1}=e_{1}, e_{1} \circ e_{2}=e_{2}, e_{2} \circ e_{1}=e_{2}, e_{2} \circ e_{2}=e_{1}$.

3. $\beta_{3}: e_{1} \circ e_{1}=e_{1}, e_{1} \circ e_{2}=e_{2}, e_{2} \circ e_{1}=e_{2}, e_{2} \circ e_{2}=0$.

4. $\beta_{4}: e_{1} \circ e_{1}=0, \quad e_{1} \circ e_{2}=0, \quad e_{2} \circ e_{1}=0, e_{2} \circ e_{2}=e_{2}$.

5. $\beta_{5}: e_{1} \circ e_{1}=e_{2}, e_{1} \circ e_{2}=0, e_{2} \circ e_{1}=0, e_{2} \circ e_{2}=0$.

6. $\beta_{6}: e_{1} \circ e_{1}=e_{1}, e_{1} \circ e_{2}=e_{2}, e_{2} \circ e_{1}=0, e_{2} \circ e_{2}=0$.

7. $\beta_{7}: e_{1} \circ e_{1}=e_{1}, e_{1} \circ e_{2}=0, e_{2} \circ e_{1}=e_{2}, e_{2} \circ e_{2}=0$.

Moreover, $\beta_{2}$ is the unique simple algebra in $\mathcal{A}^{2}$.

Remark 1. Considered over $\mathbb{C}, \beta_{1}$ and $\beta_{2}$ are isomorphic, with the change of basis given by $x_{1}=e_{1}$ and $x_{2}=i e_{2}$. Thus, we have found an example of $a$ simple associative algebra whose complexification is not simple. In $\mathcal{A}^{2}, \beta_{3}, \beta_{4}$ and $\beta_{5}$ have right and left isotropy, $\beta_{6}$ has just left isotropy and $\beta_{7}$ has just right isotropy. 
Proposition 2. Let $\beta \in \mathcal{A}^{2}$ be a two dimensional real associative algebra.

1. If $\beta$ has no isotropy, then $\beta$ is isomorphic either to $\beta_{1}$ or to $\beta_{2}$.

2. If $\beta$ has just left isotropy, then $\beta$ is isomorphic to $\beta_{6}$.

3. If $\beta$ has just right isotropy, then $\beta$ is isomorphic to $\beta_{7}$.

\section{Perturbations of associative algebras}

Considering in $\mathcal{A}^{n}$ the subspace topology induced by $\mathbb{R}^{n^{3}}$, it is possible to give the following definition:

Definition 4. An associative algebra $\beta$ is rigid in $\mathcal{A}^{n}$ if its orbit under the action of $G L(n, \mathbb{R})$ is open.

To study rigidity in the framework of Internal Set Theory, the concept of perturbation is introduced (cf. [2], [4], [6] for the details).

Definition 5. Let $n$ be standard and let $\beta_{0}$ be a real associative algebra. We say that $\beta$ is a perturbation of $\beta_{0}$ if $\beta(x, y)$ and $\beta_{0}(x, y)$ are infinitely close for all $x, y$ standard vectors of $\mathbb{R}^{n}$.

Proposition 3. A standard associative algebra law $\beta_{0} \in \mathcal{A}^{n}$ is rigid if and only if any perturbation of $\beta_{0}$ is isomorphic to it.

Proof. If $\beta_{0} \in \mathcal{A}^{n}$ is rigid, then $\mathcal{O}\left(\beta_{0}\right)$ is open. Thus, $\mathcal{O}\left(\beta_{0}\right)$ contains the halo of $\beta_{0}$ and any perturbation $\beta$ of $\beta_{0}$ is isomorphic to $\beta_{0}$. Conversely, if any perturbation belongs to $\mathcal{O}\left(\beta_{0}\right)$, then the halo of $\beta_{0}$ is contained in $\mathcal{O}\left(\beta_{0}\right)$ and, by transference, $\mathcal{O}\left(\beta_{0}\right)$ is open, so $\beta_{0}$ is rigid.

Now we enunciate a theorem, due to M. Goze [3], basic to determine the rigidity of associative algebras.

Theorem 3. Let $M_{0}$ be a standard point in $\mathbb{R}^{n}$. Then every point $M \in \mathbb{R}^{n}$ infinitely close to $M_{0}$ admits a decomposition of the form:

$$
M=M_{0}+\epsilon_{1} v_{1}+\epsilon_{1} \epsilon_{2} v_{2}+\ldots+\epsilon_{1} \epsilon_{2} \ldots \epsilon_{p} v_{p}
$$

where $\epsilon_{1}, \epsilon_{2}, \ldots, \epsilon_{p}$ are infinitely small scalars and $v_{1}, v_{2}, \ldots, v_{p}$ linearly independent vectors. Moreover, if $M=M_{0}+\eta_{1} u_{1}+\eta_{1} \eta_{2} u_{2}+\ldots+\eta_{1} \eta_{2} \ldots \eta_{q} u_{q}$ is another decomposition of $M$, then $p=q$ and the flag defined by $v_{1}, v_{2}, \ldots, v_{p}$ coincides with the flag defined by $u_{1}, u_{2}, \ldots, u_{q}$. The integer $p$ which describes the equivalence class of a point is called length of $M$. 
As a consequence of Goze's theorem, any perturbation of the standard law $\beta_{0} \in \mathcal{A}^{n}$ may be written as

$$
\beta=\beta_{0}+\epsilon_{1} \varphi_{1}+\epsilon_{1} \epsilon_{2} \varphi_{2}+\ldots+\epsilon_{1} \epsilon_{2} \ldots \epsilon_{p} \varphi_{p}
$$

where $\epsilon_{i}$ are infinitely small and $\varphi_{i}: \mathbb{R}^{n} \times \mathbb{R}^{n} \rightarrow \mathbb{R}^{n}$ are independent bilinear mappings. Considering $\beta_{0}$ a standard element of the variety and $\beta$ a perturbation of $\beta_{0}$ decomposed according to (4), we have that the shade of the straight line which joins $\beta$ and $\beta_{0}$ is a standard straight line which belongs to the tangent cone to the variety in $\beta_{0}$.

Let $\beta_{0} \in \mathcal{A}^{n}$ be a standard associative algebra law and let $f \in G L(n, \mathbb{R})$ be standard. For any $\epsilon$ infinitely small, the endomorphism $I d+\epsilon f$ belongs to the general linear group, so it makes sense to consider the action

$$
(I d+\epsilon f)^{-1} \beta_{0}((I d+\epsilon f),(I d+\epsilon f))=\beta_{0}+\epsilon\left(\delta_{\beta_{0}} f\right)+\epsilon^{2}\left(\Delta\left(\beta_{0}, f, \epsilon\right)\right),
$$

where

$$
\delta_{\beta} f(x, y)=\beta(f(x), y)+\beta(x, f(y))-f(\beta(x, y))
$$

are the 2-coboundaries of Hochschild cohomology. Moreover, since the straight line which joins $\beta_{0}$ and an infinitely close point $\beta_{0}^{\prime}$ is tangent to the orbit $\mathcal{O}\left(\beta_{0}\right)$ in $\beta_{0}$, the tangent space is given by

$$
T_{\beta_{0}} \mathcal{O}\left(\beta_{0}\right)=\left\{\delta_{\beta_{0}} f: f \in G L(n, \mathbb{R})\right\} .
$$

The dimension of the orbit is the vector dimension of $T_{\beta_{0}} \mathcal{O}\left(\beta_{0}\right)$.

Now let $\beta_{0} \in \mathcal{A}^{n}$ be a standard associative algebra and let us consider a perturbation $\beta$ of $\beta_{0}$. According to Goze's theorem, for an integer $p \leq n$, there exist $\epsilon_{1}, \ldots, \epsilon_{p}$ infinitely small, with $\epsilon_{1} \neq 0$, and $\varphi_{1}, \ldots, \varphi_{p}$ independent bilinear mappings such that:

$$
\beta=\beta_{0}+\epsilon_{1} \varphi_{1}+\epsilon_{1} \epsilon_{2} \varphi_{2}+\ldots+\epsilon_{1} \ldots \epsilon_{p} \varphi_{p}
$$

Denoting by $\beta_{1} \circ \beta_{2}$ the trilinear mapping defined as

$\beta_{1} \circ \beta_{2}(x, y, z)=\beta_{1}\left(\beta_{2}(x, y), z\right)-\beta_{1}\left(x, \beta_{2}(y, z)\right)+\beta_{2}\left(\beta_{1}(x, y), z\right)-\beta_{2}\left(x, \beta_{1}(y, z)\right)$,

$\beta$ is an associative algebra if and only if $\beta \circ \beta \equiv 0$. If the infinitesimal part is denoted by $\xi$, then $\beta=\beta_{0}+\xi$ and the latter condition is written:

$$
\beta \in A^{n} \Leftrightarrow\left(\beta_{0}+\xi\right) \circ\left(\beta_{0}+\xi\right)=\beta_{0} \circ \beta_{0}+\beta_{0} \circ \xi+\xi \circ \beta_{0}+\xi \circ \xi=0,
$$

Since $\beta_{0} \in \mathcal{A}^{n}$ and $\beta_{0} \circ \xi=\xi \circ \beta_{0}=\delta_{\beta_{0}}^{2} \xi$, we have:

$$
2 \delta_{\beta_{0}}^{2} \xi+\xi \circ \xi=0,
$$


where

$$
\delta_{\beta}^{2} \varphi(x, y, z)=\beta(\varphi(x, y), z)-\beta(x, \beta(y, z))+\varphi(\beta(x, y), z)-\varphi(x, \beta(y, z))
$$

are the 2-cocycles of Hochschild cohomology.

This is the perturbation equation of $\beta_{0}$. Developing the expression, dividing by $\epsilon_{1}$, and separating the standard and the infinitesimal part, we obtain:

$$
\begin{gathered}
\delta_{\beta_{0}} \varphi_{1}=0, \\
2\left(\epsilon_{2} \delta_{\beta_{0}}^{2} \varphi_{2}+\ldots+\epsilon_{2} \ldots \epsilon_{p} \delta_{\beta_{0}}^{2} \varphi_{p}+\epsilon_{1} \varphi_{1} \circ \varphi_{1}+\epsilon_{1} \epsilon_{2}^{2} \varphi_{2} \circ \varphi_{2}+\right. \\
\left.+\ldots+\epsilon_{1} \epsilon_{2}^{2} \ldots \epsilon_{p}^{2} \varphi_{p} \circ \varphi_{p}\right)+\epsilon_{1} \epsilon_{2} \varphi_{1} \circ \varphi_{2}+\ldots+\epsilon_{1} \ldots \epsilon_{p} \varphi_{1} \circ \varphi_{p}+ \\
+\epsilon_{1} \epsilon_{2}^{2} \epsilon_{3} \varphi_{2} \circ \varphi_{3}+\ldots+\epsilon_{1} \epsilon_{2}^{2} \ldots \epsilon_{p-1}^{2} \epsilon_{p} \varphi_{p-1} \circ \varphi_{P}=0 .
\end{gathered}
$$

\section{Rigid laws}

The interest of determining which associative algebras are rigid lies in the main role they play in the study of the variety, because their orbits are the open components of $\mathcal{A}^{n}$. In this paragraph we calculate the dimension of the orbits of the associative algebra laws obtained in the classification theorem and prove which of them are rigid. From now on, $\varphi_{i}$ will denote the Jordan algebras of theorem 1, and $\beta_{i}$ the associative algebras of theorem 2 .

Let $f \in G L(2, \mathbb{R})$, with $f\left(e_{1}\right)=a e_{1}+b e_{2}$ and $f\left(e_{2}\right)=c e_{1}+d e_{2}$, be a non-singular endomorphism. Evaluating (5) for each $\beta_{i}$, the following tangent spaces to the orbits are obtained:

$T_{\beta_{1}} f=\left(\begin{array}{cc}a & b \\ -b & a \\ -b & a \\ a-2 d & b+2 c\end{array}\right) \quad T_{\beta_{2}} f=\left(\begin{array}{cc}a & b \\ b & a \\ b & a \\ 2 d-a & 2 c-b\end{array}\right)$
$T_{\beta_{3}} f=\left(\begin{array}{ll}a & b \\ 0 & a \\ 0 & a \\ 0 & c\end{array}\right) \quad T_{\beta_{4}} f=\left(\begin{array}{cc}0 & 0 \\ 0 & b+d \\ 0 & b+d \\ -c & d\end{array}\right)$
$T_{\beta_{5}} f=\left(\begin{array}{cc}-c & 2 a-d \\ 0 & c \\ 0 & c \\ 0 & 0\end{array}\right) \quad T_{\beta_{6}} f=\left(\begin{array}{cc}a & 0 \\ 0 & a \\ c & 0 \\ 0 & c\end{array}\right) \quad T_{\beta_{7}} f=\left(\begin{array}{ll}a & 0 \\ c & 0 \\ 0 & a \\ 0 & c\end{array}\right)$

Therefore, the dimension of the orbits are:

$$
\begin{gathered}
\operatorname{dim} \mathcal{O}\left(\beta_{1}\right)=\operatorname{dim} \mathcal{O}\left(\beta_{2}\right)=4, \\
\operatorname{dim} \mathcal{O}\left(\beta_{3}\right)=\operatorname{dim} \mathcal{O}\left(\beta_{4}\right)=3, \\
\operatorname{dim} \mathcal{O}\left(\beta_{5}\right)=\operatorname{dim} \mathcal{O}\left(\beta_{6}\right)=\operatorname{dim} \mathcal{O}\left(\beta_{7}\right)=2 .
\end{gathered}
$$


To determine the open components of $\mathcal{A}^{2}$, we will apply theorem 2 and some properties of the variety of Jordan algebras. In particular, we will make use of the following theorem [1]:

Theorem 4. The only rigid algebras in $\mathcal{J}^{2}$ are $\varphi_{1}, \varphi_{2}$ and $\varphi_{6}$.

Theorem 5. The only two dimensional real associative algebras which are rigid are $\beta_{1}, \beta_{2}, \beta_{6}$ and $\beta_{7}$. Thus, $\mathcal{A}^{2}$ has two open components of dimension 4 and two open components of dimension 2.

Proof. Let $\beta_{1}=\varphi_{1}+\mu_{1}$ be, with $\varphi_{1}$ and $\beta_{1}$ the associated Jordan and Lie algebras respectively. If $\beta$ is a perturbation of $\beta_{1}$, then $\beta$ admits a decomposition of the form $\beta=\varphi+\mu$, where $\varphi \sim \varphi_{1}$ and $\mu \sim \mu_{1}$. Since $\varphi_{1}$ is rigid, there exists a basis $\left\{e_{1}, e_{2}\right\}$ such that $\varphi=\varphi_{1}$. Then, via the classification theorem (see example 1 ), $\mu \equiv 0$ and $\beta$ is isomorphic to $\beta_{1}$. An analogous reasoning proves the rigidity of $\beta_{2}$.

To prove that $\beta_{6}$ is rigid, let us consider a perturbation $\beta=\varphi+\mu$, with $\varphi \sim \varphi_{6}$. Since $\varphi_{6}$ is rigid, $\varphi$ is isomorphic to $\varphi_{6}$ and it is possible to find an infinitely close basis $\left\{x_{1}, x_{2}\right\}$, where the structure constants of $\varphi$ coincide with those of $\varphi_{6}$ and there exist $\epsilon$ and $\epsilon^{\prime}$ infinitely small such that

$$
\mu\left(x_{1}, x_{2}\right)=\epsilon x_{1}+\left(\frac{1}{2}+\epsilon^{\prime}\right) x_{2},
$$

According to our classification, the associativity condition imposes that $\epsilon=0$ and $\frac{1}{2}+\epsilon^{\prime}= \pm \frac{1}{2}$. Since $\frac{1}{2}$ and $-\frac{1}{2}$ are not infinitely close, $\epsilon^{\prime}=0$ and $\beta$ is isomorphic to $\beta_{6}$. The same reasoning proves the rigidity of the algebra $\beta_{7}$.

Now let $\beta \in \mathcal{A}^{2}$ be a standard law non-isomorphic to any of the latter algebras. Then there exists a basis $\left\{e_{1}, e_{2}\right\}$ in which the structure constants of $\beta$ are given by one of the following matrices:

$$
\left(\begin{array}{ll}
1 & 0 \\
0 & 1 \\
0 & 1 \\
0 & 0
\end{array}\right),\left(\begin{array}{ll}
0 & 0 \\
0 & 0 \\
0 & 0 \\
0 & 1
\end{array}\right),\left(\begin{array}{ll}
0 & 1 \\
0 & 0 \\
0 & 0 \\
0 & 0
\end{array}\right) .
$$

We may consider the perturbations

$$
\left(\begin{array}{ll}
1 & 0 \\
0 & 1 \\
0 & 1 \\
\epsilon & 0
\end{array}\right),\left(\begin{array}{ll}
\epsilon & 0 \\
0 & 0 \\
0 & 0 \\
0 & 1
\end{array}\right),\left(\begin{array}{ll}
0 & 1 \\
0 & 0 \\
0 & 0 \\
\epsilon & 0
\end{array}\right),
$$

all of them without isotropy. Then, by proposition 2 , these perturbations are isomorphic either to $\beta_{1}$ or to $\beta_{2}$, and the laws $\beta_{3}, \beta_{4}$ and $\beta_{5}$ are not rigid. 


\section{Contractions of associative algebras}

Using the action of the general linear group over the variety of real associative algebras, it is possible to define a formal notion of limit in $\mathcal{A}^{n}$, in analogy with the theory of contractions developed for Lie [3] and Jordan algebras [1].

Definition 6. Let $\beta_{0} \in \mathcal{A}^{n}$ be a real associative algebra and let $\left\{f_{t}\right\} \subset$ $G L(n, \mathbb{R})$ be a family of non-singular endomorphisms depending on a continous parametre $t$. If the limit

$$
\beta(x, y):=\lim _{t \rightarrow 0} f_{t}^{-1} \circ \beta_{0}\left(f_{t}(x), f_{t}(y)\right)
$$

exists for all $x, y \in \mathbb{R}^{n}, \beta$ is an associative algebra called contraction of $\beta_{0}$.

Example 2. $\beta_{3}$ is a contraction of $\beta_{1}$ by the linear transformations

$$
f_{t}\left(e_{1}\right)=e_{1}, \quad f_{t}\left(e_{2}\right)=t e_{2} .
$$

Let us consider in $\beta_{1}$ the transformed basis $\left\{x_{1}=f_{t}\left(e_{1}\right), x_{2}=f_{t}\left(e_{2}\right)\right\}$, where vector products are given by:

$$
\begin{aligned}
& x_{1} \circ x_{1}=e_{1} \circ e_{1}=e_{1}=x_{1} \\
& x_{1} \circ x_{2}=t e_{1} \circ e_{2}=t e_{2}=x_{2} \\
& x_{2} \circ x_{1}=t e_{2} \circ e_{1}=t e_{2}=x_{2} \\
& x_{2} \circ x_{2}=t^{2} e_{2} \circ e_{2}=-t^{2} e_{1}=-t^{2} x_{1}
\end{aligned}
$$

Thus, the structure constants of $\beta_{1}$ are represented by the matrix

$$
\left(\begin{array}{cc}
1 & 0 \\
0 & 1 \\
0 & 1 \\
-t^{2} & 0
\end{array}\right) .
$$

It is immediate that (7) holds for $e_{1}, e_{2}$. In the limit, an algebra isomorphic to $\beta_{3}$ is obtained. Therefore, $\beta_{3}$ is a contraction of $\beta_{1}$.

It is easy to prove from the definition that a contraction of $\beta_{0}$ corresponds to a closure point of $\mathcal{O}\left(\beta_{0}\right)$. In particular, rigid algebras are not obtained as a contraction of any non-isormorphic algebra in $\mathcal{A}^{n}[7]$. It is obvious that the change of basis $x_{i}=t e_{i}, i=1, \ldots, n$ induces a contraction of any associative algebra over the Abelian algebra. Moreover, for every contraction the following inequality holds:

$$
\operatorname{dim} \mathcal{O}\left(\beta_{0}\right)>\operatorname{dim} \mathcal{O}(\beta)
$$

That gives us a first criterion to study the contractions of the variety. If $\beta$ is a contraction of $\beta_{0}$, then its associated Jordan algebra $\varphi$ is also a contraction of $\varphi_{0}$. We have already proved [1] that $\beta_{4}$ is not a contraction of $\beta_{1}$ in $\mathcal{J}^{2}$. The following theorem specifies how to obtain the remaining contractions. 
On the variety of two dimensional real associative algebras

Theorem 6. Let $\beta_{i}$ be the associative algebras of the classification theorem. Then $\beta_{3}, \beta_{4}$ and $\beta_{5}$ are the only algebras in $\mathcal{A}^{2}$ which appear as the contraction of an associative algebra. More precisely:

1. $\beta_{3}$ is a contraction of $\beta_{1}$ and $\beta_{2}$.

2. $\beta_{4}$ is a contraction of $\beta_{2}$.

3. $\beta_{5}$ is a contraction of $\beta_{1}, \beta_{2}, \beta_{3}$ and $\beta_{4}$

Proof. Since $\beta_{1}, \beta_{2}, \beta_{6}$ and $\beta_{7}$ are rigid algebras, they are not contractions of any other associative algebra.

1. We have already proved that when we consider the family of non-singular endomorphisms

$$
f_{t}\left(e_{1}\right)=e_{1}, \quad f_{t}\left(e_{2}\right)=t e_{2},
$$

a contraction of $\beta_{1}$ over $\beta_{3}$ is obtained. The same family of linear transformations defines the contraction of $\beta_{2}$ over $\beta_{3}$.

2. Considering the parametric change of basis

$$
f_{t}\left(e_{1}\right)=t e_{1}, \quad f_{t}\left(e_{2}\right)=\frac{1}{2}\left(e_{1}+e_{2}\right),
$$

the limit when $t \longrightarrow 0$ gives an algebra isomorphic to $\beta_{4}$.

3. When considered in $\beta_{1}$, the family of linear transformations

$$
f_{t}\left(e_{1}\right)=\sqrt{\frac{t}{2}}\left(e_{1}+e_{2}\right), \quad f_{t}\left(e_{2}\right)=t e_{2}
$$

defines an algebra isomorphic to $\beta_{5}$ in the limit.

The parametric change of basis

$$
f_{t}\left(e_{1}\right)=t e_{2}, \quad f_{t}\left(e_{2}\right)=t^{2} e_{1}
$$

induce a contraction of $\beta_{2}$ over $\beta_{5}$.

In the same way, if we take the family of non-singular endomorphisms

$$
f_{t}\left(e_{1}\right)=t\left(e_{1}+e_{2}\right) \quad f_{t}\left(e_{2}\right)=t^{2} e_{2},
$$

a contraction of $\beta_{3}$ over $\beta_{5}$ is obtained.

Finally, if we apply to $\beta_{4}$ the linear transformations

$$
f_{t}\left(e_{1}\right)=e_{1}+t e_{2}, \quad f_{t}\left(e_{2}\right)=t^{2} e_{2},
$$

in the limit when $t \longrightarrow 0$, it comes that $\beta_{5}$ is a contraction of $\beta_{4}$. 
We summarize the classification and the contractions obtained for two dimensional real associative algebras in the following diagram, where each contraction is represented by an arrow. One can verify that rigid algebras correspond to those matrices which do not receive any arrow.

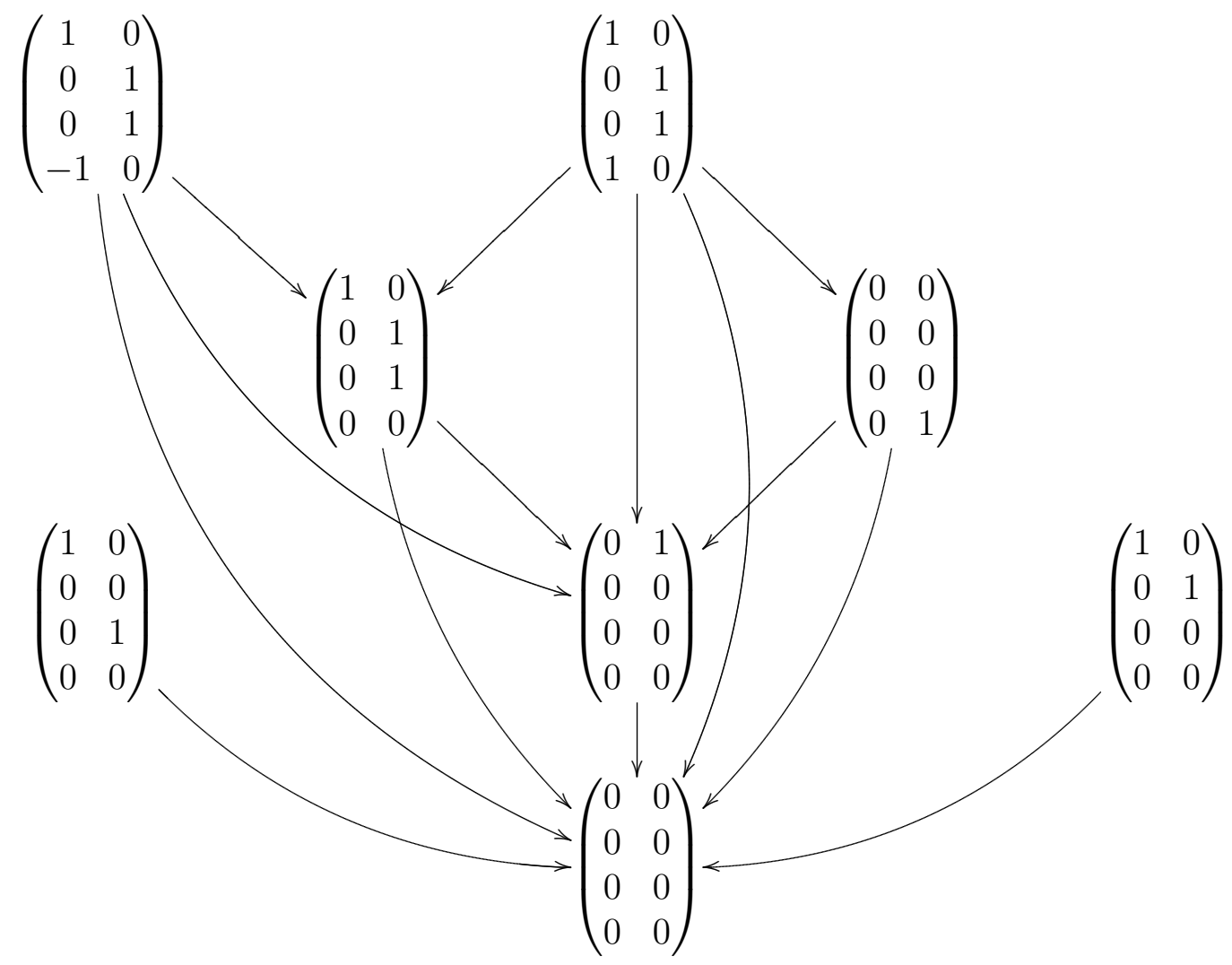

\section{References}

[1] J. M. Ancochea Bermúdez, R. Campoamor-Stursberg, L. García Vergnolle, J. Sánchez Hernández, Contractions d'algèbres de Jordan en dimension 2, to appear in Journal of Algebra.

[2] F. Diener, G. Reeb. Analyse Non Standard, Hermann, 1989.

[3] M. Goze. Etude local de la variété des lois d'algèbres de Lie, Thèse d'état, Mulhouse, 1982.

[4] R. Lutz, M. Goze. Non standard analysis. A practical guide with applications. Lect. Notes in Math. 881. Springer-Verlag, 1981. 
[5] A. Makhlouf. Sur les algèbres associatives rigides, Thèse, Mulhouse, 1990.

[6] E. Nelson. Internal Set Theory: a new approach to Nonstandard Analysis, Bull. Amer. Math. Soc. 83 (1977), 1165-1198.

[7] A. Nijenhuis, R. W. Richardson. Cohomology and deformation of algebraic structures, Bull. Amer. Math. Society 70 (1964), 406-411.

[8] A. Robinson. Non standard analysis. North Holland, 1966.

Received: May 14, 2007 